\title{
Degradation Behavior and Immunological Detection of Silk Fibroin Exposure to Enzymes
}

\author{
Ruru Chen,* Lian Zhou,* Hui YANG, ${ }^{*}$ Hailing Zheng, ${ }^{*}$ Yang ZHOU, ${ }^{* *}$ Zhiwen HU,*** and \\ Bing WANG ${ }^{* \dagger}$ \\ *Key Laboratory of Advanced Textile Materials and Manufacturing Technology, Ministry of Education, \\ Zhejiang Sci-Tech University, Hangzhou 310018, China \\ **Key Scientific Research Base of Textile Conservation, State Administration for Cultural Heritage, \\ China National Silk Museum, Hangzhou 310002, China \\ ***Institute of Textile Conservation, Zhejiang Sci-Tech University, Hangzhou 310018, China
}

\begin{abstract}
The degradation behavior of silk fibroin (SF) is a significant and intriguing subject in the area of archaeological ancient silk research. In the present study, the immunological detection techniques combined with traditional characterization methods, jointly studied the degradation process of SF from Bombyx mori (B. mori) and Antheraea pernyi (A. pernyi) through exposure to alkaline proteinase, $\alpha$-chymotrypsin, pepsin, and trypsin. Spectroscopic analysis revealed that different enzymes showed similar hydrolysis effects on the secondary structure, but the changes of $B$. mori SF and $A$. pernyi SF were mainly reflected in the decrease of $\beta$-sheet and the reduction of $\alpha$-helical structure, respectively. In further research of immunology, two diagnostic antibodies were prepared corresponding to SF of B. mori and A. pernyi, respectively. The enzyme-linked immunosorbent assay (ELISA) and western blot indicated the enzyme-treated SF proteins still exhibited higher immunoreactivity because the epitopes on the surface of SF molecules are retained. Although $\alpha$-chymotrypsin possesses the most cleavage sites among these enzymes, the $\alpha$-chymotrypsin-treated SF did not exhibit significant changes in secondary structures and high antibody binding capacity. The results deepen our understanding of the SF degradation process during enzymatic hydrolysis, and show far-reaching guiding significance in trace detection of SF.
\end{abstract}

Keywords Silk fibroin, enzymes, degradation, immunoassay

(Received June 13, 2019; Accepted July 17, 2019; Advance Publication Released Online by J-STAGE July 26, 2019)

\section{Introduction}

As a nature polymer, silk is one of the most indispensable material in the long history of human beings with great economic and practical value, as well as an important part of ancient Chinese civilization. Many studies proved that silk originated in China thousands of years ago, and was introduced to the Middle East and Europe as a well-known luxury commodity. ${ }^{1,2}$ In recent decades, more and more silk fabrics made of silk fibroin (SF) from B. mori and A. pernyi were unearthed..$^{3-5}$ But their degradation is a high-profile problem because the deterioration of archaeological silk is determined by the burial environment of soil composition, temperature, $\mathrm{pH}$, impurities and oxygen contents, especially microorganism. ${ }^{6}$ This is because the enzymes secreted by bacteria and fungi cause severe degradation of precious cultural heritage. ${ }^{7,8}$ Meanwhile, silk is the source of nutrients $(\mathrm{C}, \mathrm{N}$ and $\mathrm{S}$ ) for the growth and reproduction of microorganisms, thereby forming a vicious circle, which accelerates the breaking of macromolecular chains and degraded into small molecules of polypeptides or amino acids.

$\dagger$ To whom correspondence should be addressed. E-mail: wbing388@163.com (B.W.); cnsmzhouyang@126.com (Y. Z.)
Several studies have analyzed the degradation behavior of regenerated silk fibroin. Lu et al. ${ }^{9}$ focused on regenerated fibroin film exposed to protease XIV solution, and found that the higher is the $\beta$-sheet content, the faster is the degradation rate. In addition, the $3 \mathrm{D}$ scaffolds, hydrogels and nanoparticles made by regenerated silk fibroin have also been discussed..$^{10,11}$ Nevertheless, the degradation process of natural silk fibroin is more complicated and bewildering because of the complex structure. Different amino acid sequences fold into secondary structures of random coil, $\alpha$-helix and $\beta$-sheet conformation. The $\beta$-sheets form the crystalline region, which was confirmed as a rectangular unit cell containing four molecular with several parameters: $a$ (interchain direction) $=9.38 \AA, b$ (intersheet direction) $=9.49 \AA$ and $c$ (fiber axis) $=6.98 \AA$ in fibroin of B. mori. ${ }^{12}$ The tertiary structure is maintained by hydrophobic interactions between amino acid side chains, hydrogen bonds, van der Waals forces and electrostatic interactions. ${ }^{13}$ Therefore, it is necessary to pay more attention to understand the degradation process and mechanism of natural silk fibroin.

Additionally, the antigenicity (the ability of antigens to bind specifically to the antibodies they induce) of degraded silk fibroin is another focus of attention on account of the great significance in the detection of archaeological fabrics. ${ }^{14}$ After thousands of years of degradation, it is difficult to find physical evidence to prove the existence of silk. Therefore, the 

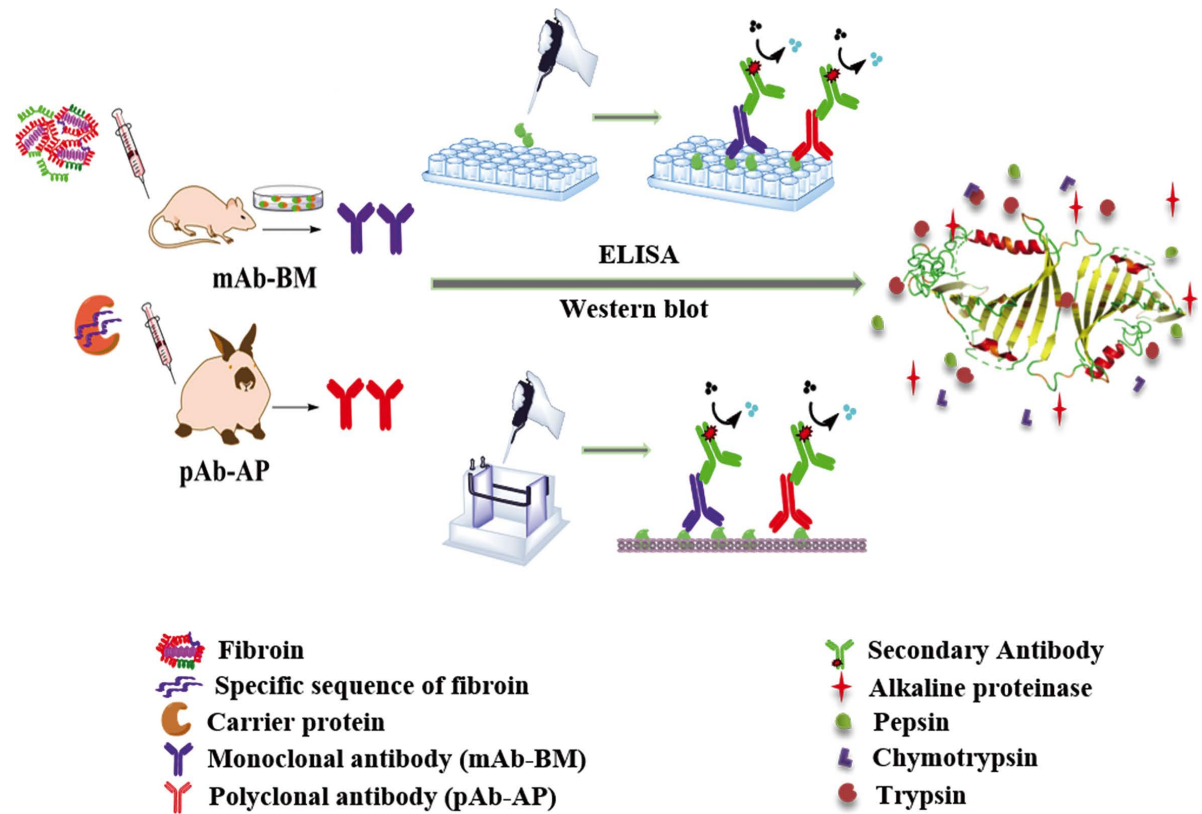

Fig. 1 Schematic illustration of ELISA and western blot for the detection of B. mori and A. pernyi silks.

immunological detection technology, such as enzyme-linked immunosorbent assay (ELISA) and immunoblotting, ${ }^{3,15}$ are performed to detect the target protein, the degraded silk fibroin. These immunological detection methods are extensively used for biochemistry studies owing to the advantages of great specificity, high sensitivity and low sample consumption. Meanwhile, they can provide accurate and reliable diagnostic information through antigen-antibody reactions.

In this study, we explored the degradation behavior of silk fibroin in B. mori and A. pernyi following immersion in enzyme (alkaline proteinase, $\alpha$-chymotrypsin, pepsin, and trypsin) solutions by determining the structure and molecular weight distribution. Further, specific antibodies were generated using fibroin (from B. mori) or the diagnostic sequence (from A. pernyi) conjugated with carrier protein as the immunogen for animal immunization. The antigenicity (binding ability to diagnostic antibodies) of the degraded silk fibroin from $B$. mori and $A$. pernyi was examined with the corresponding diagnostic antibodies by ELISA and western blot assay (Fig. 1). This study has enabled us to provide a better understanding of the changes in amino acid composition and protein structure, especially the immunological properties of degraded silk fibroin, during the enzymatic hydrolysis process.

\section{Experimental}

\section{Reagents}

Goat anti-rabbit secondary conjugated to horseradish peroxidase (goat anti-rabbit IgG-HRP antibody), Freund's complete adjuvant, Freund's incomplete adjuvant, and saline were supplied by Hangzhou Hua'an Biotechnology Co., Ltd. Sodium carbonate, sodium chloride, potassium chloride, sodium hydroxide, disodium hydrogen phosphate, potassium dihydrogen phosphate, hydrogen chloride, and methanol were provided by Hangzhou Gaojing Fine Chemical Co., Ltd. Albumin bovine serum (BSA) and alkaline proteinase from Bacillus licheniformis were purchased from Beijing Solarbio Science \& Technology
Co., Ltd. $\alpha$-Chymotrypsin from bovine pancreas, pepsin from porcine gastric mucosa, and trypsin from bovin pancreas were purchased from Aladdin (Los Angeles, southern California). Sodium dodecyl sulfate (SDS), bromophenol blue, dithiothreitol, glycine, glycerol, Tris(hydroxymethyl)aminomethane, Tris(hydroxymethyl)aminomethane hydrochloride, $N, N, N^{\prime}, N^{\prime}$-tetramethylethylenediamine, isopropyl alcohol, ammonium persulphate, and Tween-20 were also purchased from Aladdin. Cocoons of $B$. mori and $A$. pernyi were provided by China National Silk Museum.

\section{Preparation of the specific anti-SF primary antibody}

For $B$. mori, the fibroin was selected as the complete antigen for immunization to produce a diagnostic monoclonal antibody (mAb-BM). The protocols of producing the primary antibody were narrated in our previous report. ${ }^{16}$ The concentration of $\mathrm{mAb}-\mathrm{BM}$ was $1.0 \mathrm{mg} / \mathrm{mL}$.

In parallel, the species-specific amino acid sequence "CSHSHSYEASRISVH" of A. pernyi SF was determined as the hapten, which was synthesized and coupled with the keyhole limpet hemocyanin (Sangon, Shanghai, China) to generate the complete antigen. Two New Zealand white rabbits, 14-16 weeks old, were injected with the immunogen, which consisted of $500 \mu \mathrm{g}$ of the complete antigen diluted in saline and 1:1 mixed with Freund's complete adjuvant, and ear blood samples were drawn before immunization as control samples. After the primary immunization with $100 \mathrm{~mL}$ of immunogen, three subsequent injections were carried out to boost the immunization at 2-week intervals using Freund's incomplete adjuvant instead of Freund's complete adjuvant. Ten days after the third and fourth immunizations, the rabbits were ear-bled, and the serum titers of the blood samples were tested by indirect ELISA. Sera were collected to obtain the antibody (pAb-AP) once the titer reached the required value, the pAbs were purified and stored $(0.33 \mathrm{mg} / \mathrm{mL})$ at $-20^{\circ} \mathrm{C}$ until use.

\section{Indirect enzyme linked immunosorbent assay (ELISA)}

Indirect ELISA was carried out on a 96-well plate. Each well was coated with $100 \mu \mathrm{L}$ of fibroin powders $(100 \mu \mathrm{g} / \mathrm{mL})$ in $\mathrm{CB}$ 
(carbonate buffer, $\mathrm{pH} 9.6$ ) and incubated at $4{ }^{\circ} \mathrm{C}$ overnight. After the solution was decanted, the coated plate was washed with $200 \mu \mathrm{L}$ of PBS (phosphate-buffered saline, $\mathrm{pH}$ 7.4) three times. To eliminate the remaining active spots, the blocking buffer (200 $\mu \mathrm{L} /$ well) was added and incubated at $37^{\circ} \mathrm{C}$ for $2 \mathrm{~h}$, and the plate was then washed again. After $100 \mu \mathrm{L}$ of diagnostic primary antibody (diluted 1:1000) was added to each well and incubated at $37^{\circ} \mathrm{C}$ for $1 \mathrm{~h}$, unbound compounds were removed by washing with PBS. A total of $100 \mu \mathrm{L}$ of the secondary antibody (diluted 1:2000) was subsequently added at $37^{\circ} \mathrm{C}$ for another $1 \mathrm{~h}$, followed by washing five times with PBS. An aliquot of $100 \mu \mathrm{L}$ TMB was added to the plates in the dark for $10 \mathrm{~min}$ at $37^{\circ} \mathrm{C}$ and subsequently $100 \mu \mathrm{L}$ of stop solution was added to terminate the enzymatic reaction. The optical density of samples was read at $450 \mathrm{~nm}\left(\mathrm{OD}_{450}\right)$ using a microplate reader (550, Bio-Rad).

Several controls were employed as follows to confirm the accuracy: silk fibroin as the positive control, $\mathrm{CB}$ as the negative control, and the primary antibody replaced with blocking buffer as the blank control. Meanwhile, five parallel wells were utilized for each sample and control.

The cutoff was the mean $\mathrm{OD}_{450}$ of negative control $(\mathrm{CB})$ plus its three standard deviations. Therefore, the $\mathrm{OD}_{450}$ values below and above the cutoff were considered as negative and positive for ELISA test, respectively.

\section{Western blot analysis}

Immunoblotting was performed similar to the methods described in previous reports. ${ }^{17}$ The proteins separated by SDSPAGE were transferred to a PVDF membrane at a constant current of $200 \mathrm{~mA}$ for $1.5 \mathrm{~h}$ using a Mini Trans-Blot Cell (Bio-Rad, USA). The membrane was blocked with a blocking solution for $1 \mathrm{~h}$ to prevent nonspecific binding. Then, the membrane was incubated with the primary antibody (diluted 1:1000) in PBST at room temperature. After incubation for $1 \mathrm{~h}$ on a shaker, the membrane was washed three times (10 min per time) in $20 \mathrm{~mL}$ PBST, and then it was incubated with the secondary antibody (diluted 1:2000) for another hour. After three washes with PBST, the immunoreactive sites of the blots were detected with Clarity Western ECL substrate (Bio-Rad, USA).

\section{Results}

Morphology

The morphological changes of silk fibers during the enzymolysis process were as shown in Figs. $2 \mathrm{a}-2 \mathrm{j}$. The untreated silk fibroin of B. mori (Fig. 2a) and A. pernyi (Fig. 2f) had a relatively smooth surface, except for the longitudinal striations and little burrs on the surface of the latter. However, all remaining silk fibroin after treatment with alkaline proteinase (SF-A), $\alpha$-chymotrypsin (SF-C), pepsin (SF-P) and trypsin (SF-T) showed damage with extensive longitudinal burrs, splits, and even substantial cleavages, especially for B. mori SF-C and SF-T (Figs. 2c, 2e), which suggested an enzymatic attack of the fibroins. Since the enzymatic hydrolysis was relatively mild, the degree of enzymatic hydrolysis of silk fibroin by different enzymes cannot be easily distinguished from the surface morphology and required further analysis.

\section{Secondary structure}

The secondary structure of silk fibers was assessed by XRD (Fig. S1) and ATR-FTIR (Fig. S2). The amide III region $\left(1180-1300 \mathrm{~cm}^{-1}\right)$ was chosen to fitting peaks to analyze the content $(\%)$ of different structure. Generally, the amide III was
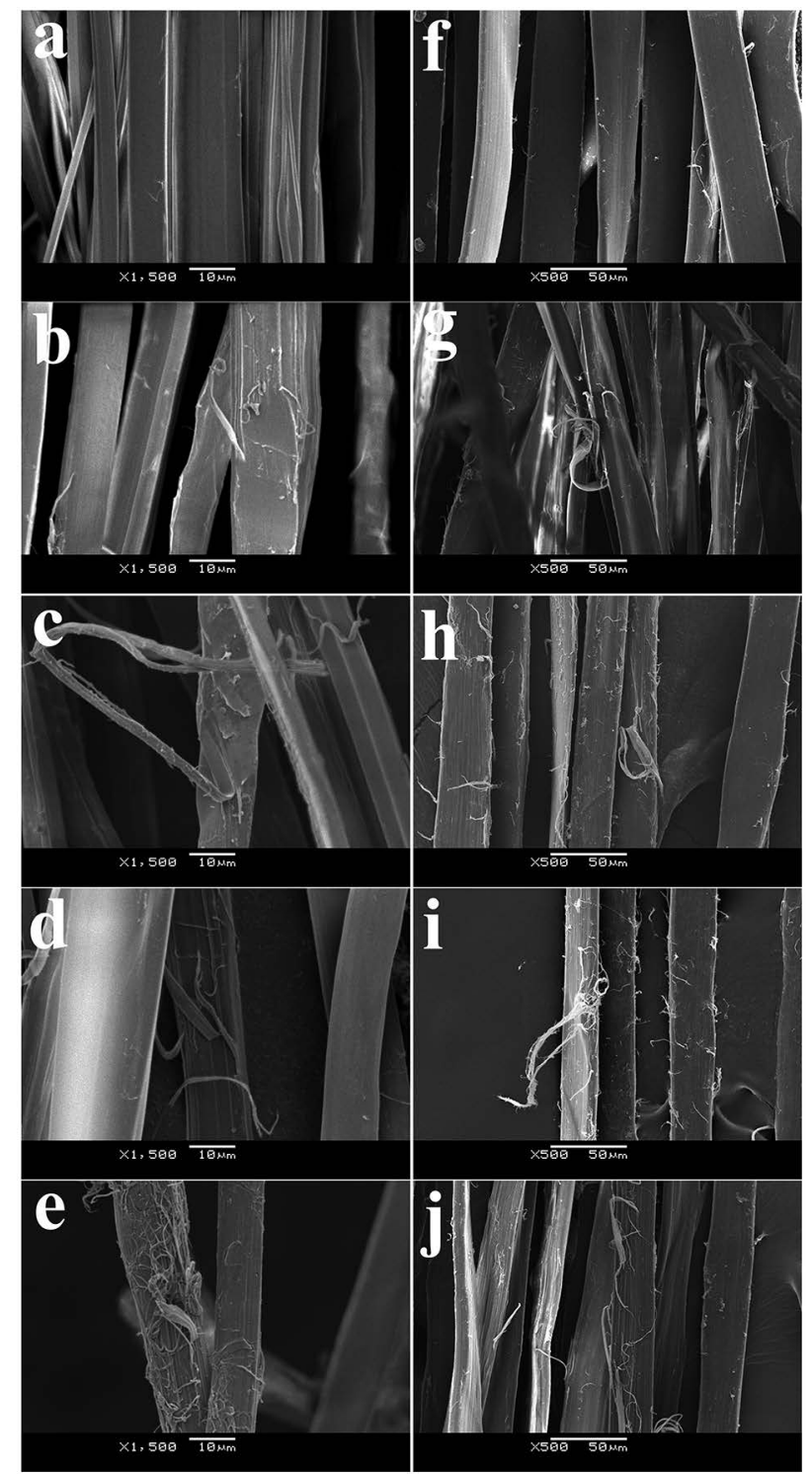

Fig. 2 SEM images of silk fibers of B. mori and A. pernyi. (a) Untreated silk fibers of $B$. mori, (b), (c), (d) and (e) treated silk fibers by alkaline proteinase, chymotrypsin, pepsin and trypsin, respectively; (f) untreated silk fibers of A. pernyi, (g), (h), (i) and (j) treated SF by alkaline proteinase, chymotrypsin, pepsin and trypsin, respectively.

identified as follows: for B. mori, $1232 \mathrm{~cm}^{-1}$ as random coil $/ \alpha$-helical structure and $1265 \mathrm{~cm}^{-1}$ as $\beta$-sheet structure; for A. pernyi, $1222 \mathrm{~cm}^{-1}$ as $\beta$-sheet conformation, $1240 \mathrm{~cm}^{-1}$ as random coil, and $1265 \mathrm{~cm}^{-1}$ as $\alpha$-helix. ${ }^{18,19}$ As shown in Fig. 3, enzymolysis decreased the content of $\beta$-sheet conformation $(28.2-29.3 \%)$ in B. mori silk fibroin compared to the $\beta$-sheet content of $30.8 \%$ in the untreated protein. The decrease in $\beta$-sheets was the result of the destruction of ordered regions by enzymes. In parallel, enzymes significantly reduced the $\alpha$-helix structure of $A$. pernyi, and the silk fibroin treated with pepsin had the lowest $\alpha$-helix content of $21.84 \%$, which led to a proportional increase in random coils and $\beta$-sheets. The semiquantitative analysis implied that enzymes degraded the $\beta$-sheet and $\alpha$-helix structure in $B$. mori and A. pernyi, respectively.

\section{Protein isolation and distribution}

The product of enzymolysis was then investigated by SDS- 
PAGE to show the separation and distribution of fibroin. The molecular weights (MW) of the four enzymes were characterized as controls at the outset to remove the protease interference. As shown in Fig. 4a, alkaline proteinase had no obvious bands while $\alpha$-chymotrypsin, pepsin and trypsin had strong bands in different ranges. Fortunately, all of the bands did not appear in silk fibroin samples in Figs. 4b, 4c, revealing that the enzymes did not interfere with the molecular-weight distribution of silk fibroin.

In contrast, all enzyme-treated silk fibroin of B. mori showed

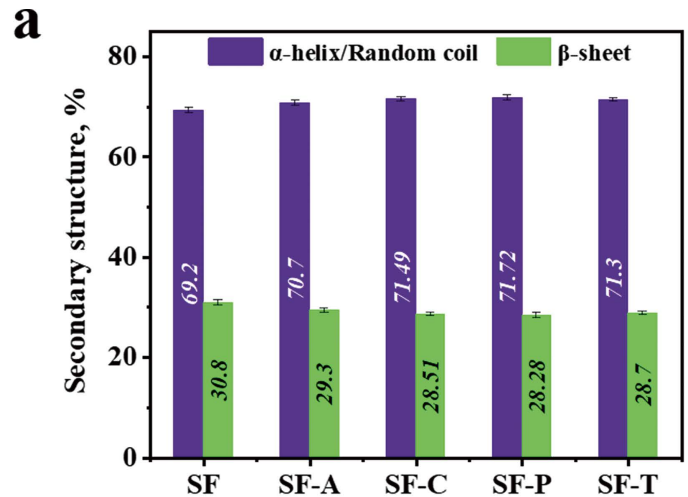

b

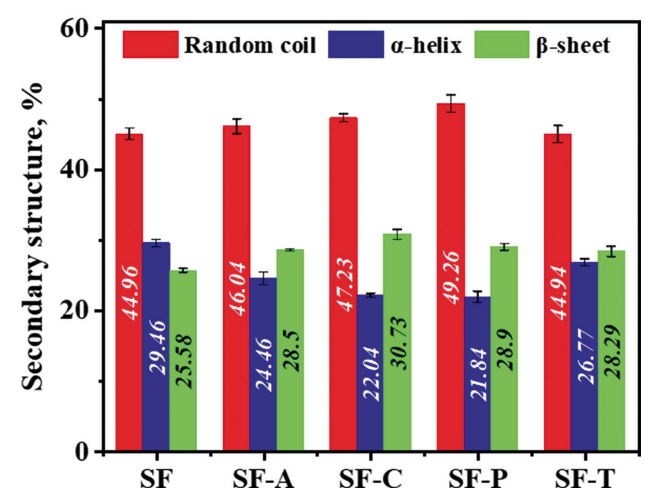

Fig. 3 Quantification of the secondary structure (\%) of silk fibroin before and after enzymatic hydrolysis. (a) B. mori, (b) A. pernyi. Untreated silk fibroin (SF); treated by alkaline proteinase (SF-A); treated by chymotrypsin (SF-C); treated by pepsin (SF-P); treated by trypsin (SF-T). a diffuse distribution in the whole lane and was concentrated below $20 \mathrm{kDa}$, compared to the control silk fibroin. In addition, a smeared band at $40 \mathrm{kDa}$ was detected in silk fibroin degraded by trypsin. However, the native silk fibroin of $B$. mori consisted of a $350-\mathrm{kDa}$ heavy chain (H-chain), a 26-kDa light chain (L-chain) and a 30-kDa fibrohexamerin (Fhx, or P25) with a 6:6:1 molar ratio. ${ }^{1,20,21}$ The experimental and theoretical differences in the MW demonstrated that the enzymes degraded the silk fibroin macromolecules into smaller peptides. In parallel, the SDS-PAGE analysis of silk fibroin from A. pernyi showed a similar distribution and concentration to those of B. mori. Likewise, there was a small band at approximately $40 \mathrm{kDa}$ in silk fibroin degraded by alkaline proteinase, $\alpha$-chymotrypsin and trypsin that was absent in silk fibroin degraded by pepsin. The difference in the molecular weight distribution of different samples indicated that silk fibroin molecular chains were degraded via enzymes to different levels.

\section{ELISA of enzyme-treated silk fibroin}

Silk fibroin digested by different enzymes was identified and analyzed via indirect ELISA that we established in our previous work. $^{22}$ To estimate the specificity of the assay, the crossreactivity of the antibodies was tested with the four enzymes used in this work, including alkaline proteinase, $\alpha$-chymotrypsin, pepsin and trypsin. As shown in Figs. 5a and 5b, there were no positive values appearing except for the positive control (silk fibroin), revealing that the antibodies had high specificity and did not bind to the enzyme. Based on the results, we confirmed that the anti-SF antibodies could be applied to detect silk fibroin while eliminating the interference of the enzymes.

A total of four SF samples treated with enzymes and three controls were subjected to ELISA; the results are shown in Figs. $5 \mathrm{c}$ and $5 \mathrm{~d}$. All of the enzyme-treated samples were positive compared to the blank and negative controls, meaning that the diagnostic antibodies could specifically identify the silk fibroin obtained by enzymatic hydrolysis, further indicating that the antigenic epitope of the SF protein maintained a certain integrity for immunological identification. Interestingly, the OD values greatly differed among the testing samples. Among the samples analyzed in this study, the silk fibroin treated by pepsin had the highest values, followed by alkaline proteinase and trypsin, which may be related to differences in the cleavage sites of different enzymes.

a

b

C

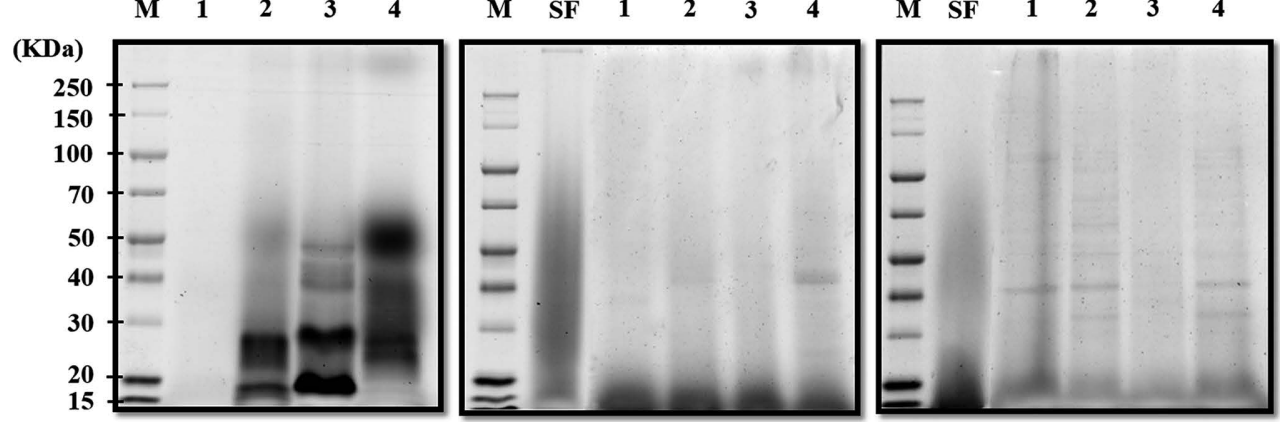

Fig. 4 The SDS-PAGE of (a) enzymes and silk fibroin of (b) B. mori and (c) A. pernyi. Lane M, Molecular weight marker; lane SF, the untreated silk fibroin; lane 1, degraded by alkaline proteinase; lane 2 , degraded by chymotrypsin; lane 3 , degraded by pepsin; lane 4 , degraded by trypsin. 
$\mathbf{a}$

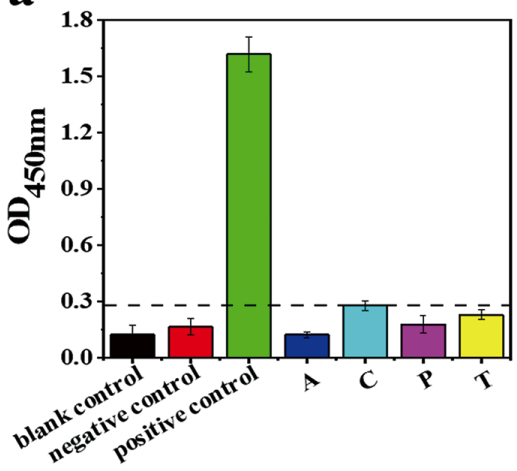

c

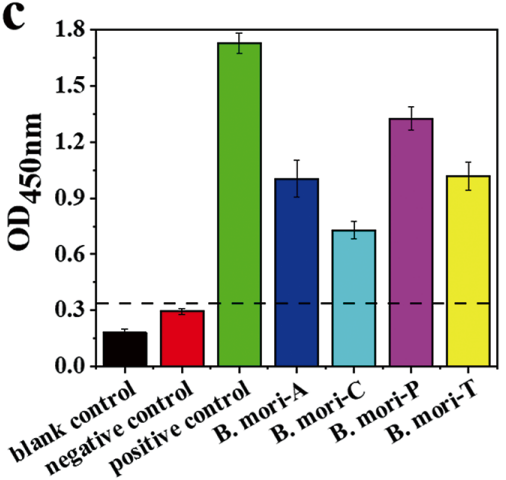

b

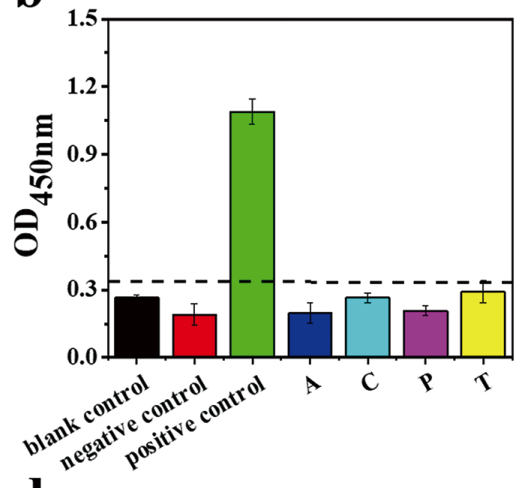

d

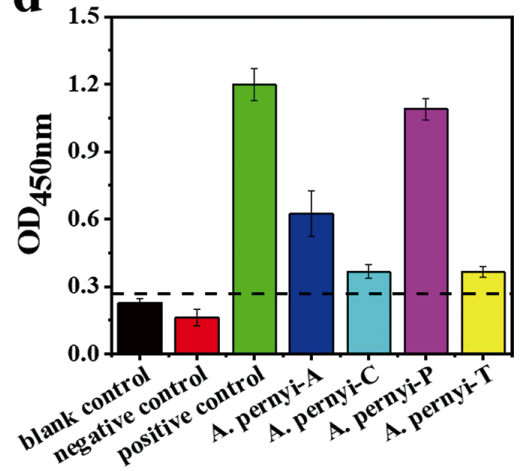

Fig. 5 ELISA results with diagnostic antibody: (a) ELISA results of enzymes with mAb-BM, (b) ELISA results of enzymes with pAb-AP, (c) ELISA results of silk fibroin from $B$. mori degraded by enzymes with mAb-BM, (d) ELISA results of silk fibroin from A. pernyi degraded by enzymes with pAb-AP. The dashed line refer to the cutoff value.

$\mathbf{a}$

b

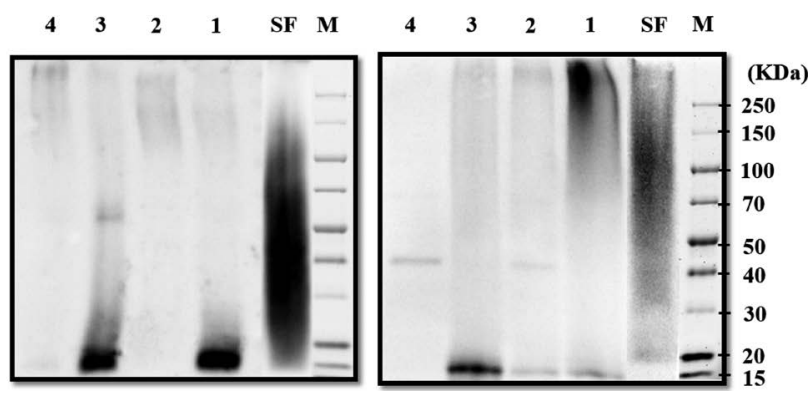

Fig. 6 Western blot assay of silk fibroin of (a) B. mori and (b) A. pernyi with $\mathrm{mAb}-\mathrm{BM}$ and $\mathrm{pAb}-\mathrm{AP}$, respectively. Lane M, Molecular weight marker; lane SF, the untreated silk fibroin; lane 1, degraded by alkaline proteinase; lane 2 , degraded by chymotrypsin; lane 3 , degraded by pepsin; lane 4 , degraded by trypsin.

\section{Immunoblotting of enzyme-treated $S F$ protein}

To further validate the effectiveness of specific antibodies for silk fibroin detection, western blot were performed on four enzyme-treated samples of B. mori and A. pernyi. As observed in Fig. $6 a$, the antibody of mAb-BM specifically bound to all samples, which again confirmed that the diagnostic antibody of mAb-BM could be used to detect silk fibroin with high sensitivity, although the silk fibroin is enzymatically hydrolyzed into small polypeptide molecules. Nevertheless, strong antibody binding to a protein with a MW of $15 \mathrm{kDa}$ was observed in silk fibroin degraded by alkaline proteinase and pepsin. By comparison, only the distributed bands having MWs values above $100 \mathrm{kDa}$ in samples degraded by $\alpha$-chymotrypsin and trypsin were observed. The results were in accordance with ELISA, suggesting that the SF protein obtained by alkaline proteinase and pepsin had a higher antibody-binding capacity.

The diagnostic pAb-AP antibody was applied to a western blot, as shown in Fig. 6b. There was a small band of approximately $15 \mathrm{kDa}$ in silk fibroin degraded by alkaline proteinase, $\alpha$-chymotrypsin, but a strong band appeared in silk fibroin protein degraded by pepsin. Conversely, the signal intensity of a smear of protein with MWs above $70 \mathrm{kDa}$ in silk fibroin degraded by alkaline proteinase was significantly higher than that degraded by $\alpha$-chymotrypsin and pepsin. Besides, only a small band of approximately $40 \mathrm{kDa}$ was present in silk fibroin degraded by trypsin. These results indicted the different antibody-binding capacities of silk fibroin obtained via different enzymes. Combined with the western blotting results of B. mori, we concluded that alkaline proteinase and pepsin were more effective than other enzymes for hydrolyzing silk fibroin.

\section{Discussion}

The serine proteases (alkaline proteinase, $\alpha$-chymotrypsin and trypsin) and aspartic protease (pepsin) are typical endopeptidases that were selected to hydrolyze the silk fibroin. The former uses a catalytic serine in its active site to cleave peptide bonds continuous to aliphatic, aromatic or hydrophobic residues. Similarly, the latter uses a catalytic aspartate in its active site to efficiently cleave peptide bonds between hydrophobic and preferably aromatic amino acids such as phenylalanine, 
tryptophan, and tyrosine..$^{11,23,24}$ The predicted cleavage sites of the four enzymes within SF are given in Table 1. It should not be surprising that there would be a signification difference in experimental results, given that each enzyme has a distinctive kind and number of cleavage sites.

In terms of the surface morphology, all remaining silk fibers still kept the fiber-like structures in SEM images (Fig. 2) after enzymatic treatment, and it was difficult to distinguish the degree of enzymatic hydrolysis because the destruction on silk fibers via enzymes was determined by the limited efficiency and experimental conditions. However, the silk fibroin obtained from the solution was degraded via enzymes so severely that the molecular weight migrated under $20 \mathrm{kDa}$ (Fig. 4). The above results indicated that the destruction of silk fibers by enzymatic hydrolysis might be a gradual process from the outside to the inside.

Referring to the secondary structure, the L-chain and P25 are hydrophilic, constituting the amorphous domains, while the $\mathrm{H}$-chain is hydrophobic and predominantly consists of the repeating GAGAGS and GAGAGY motifs, which form $\beta$-sheet as the crystalline region in $B$. mori SF. $^{25,26}$ However, only the $\mathrm{H}$-chain is found in A. pernyi SF and it is characterized by Gly-rich domains and repetitive poly(Ala) motifs. ${ }^{26,27}$ Differences in the amino acid sequences perhaps explained why the degradation of the secondary structure of SF between B. mori and A. pernyi was distinguishing. We expected that most of the evident changes in the secondary structure were induced by $\alpha$-chymotrypsin because it had the most recognized sites. While $\alpha$-chymotrypsin was only degraded in less crystalline regions and had no significant effect on the $\beta$-sheet structure. ${ }^{12}$ Simultaneously, the XRD (Fig. S1) and ATR-FTIR spectra (Fig. S2) revealed an unexpected finding that all of the enzymes showed similar decreasing trends in the degradation of the $\beta$-sheet of $B$. mori SF in spite of the different specificities and cleavage sites. One explanation was that the hindrance of the secondary structure in regard to the accessibility of the recognition sites was not considered in the calculation of the cleavage sites. Unlike the ordered $\beta$-sheet structure, the $\alpha$-helix conformation of $A$. pernyi SF was conspicuously decreased because the swelling of the unordered regions enabled the proteases to access to the cleavage sites.

In immunoassay, it is well-known that the antibody-binding capacity is directly related to epitopes, the part of an antigen that is recognized specifically by antibodies, containing linear and conformation epitopes. ${ }^{28}$ Linear epitopes, also known as continuous epitopes, are composed of sequentially consecutive amino acids on the peptide chain. Conformational epitopes, also known as discrete epitopes, are composed of amino acids that are spatially adjacent but discontinuous in sequence. Due to the different cleavage sites, the number of epitopes on the molecular chain of the obtained SF was different, resulting in a different binding ability to the diagnostic antibody. For the SF degraded by trypsin, the efficiency of enzymatic hydrolysis was not satisfactory compared to other samples because of much fewer cleavage sites (Table 1), further resulting in the less epitopes binding to the diagnostic antibodies, which explained the lower $\mathrm{OD}_{450}$ in ELISA and the distributed bands in western blot. Differently, in the case of SF samples degraded by $\alpha$-chymotrypsin, the epitopes on the molecular chain was able to be destroyed owing to the numerous cleavage sites. For instance, among the diagnostic sequence of CSHSHSYEASRISVH from A. pernyi used to prepare the antibody, three amino acids including tyrosine $(\mathrm{Y})$, isoleucine (I) and valine (V) were the cleavage sites of $\alpha$-chymotrypsin, leading to the destruction of epitopes, which might be an
Table 1 Summary of the predicted cleavage sites of protease in silk fibroin

\begin{tabular}{|c|c|c|c|c|c|}
\hline \multirow{3}{*}{ Protease } & \multirow{3}{*}{$\begin{array}{l}\text { Cleavage } \\
\text { sites }\end{array}$} & \multicolumn{4}{|c|}{ Number of cleavage sites } \\
\hline & & \multicolumn{3}{|c|}{ B. mori SF } & \multirow{2}{*}{$\frac{\text { A. pernyi SF }}{\text { Fibroin }}$} \\
\hline & & $\mathrm{HC}$ & $\mathrm{LC}$ & P25 & \\
\hline Alkaline proteinase & $\begin{array}{l}\text { Glu, Met, } \\
\text { Leu, Tyr, } \\
\text { Lys }\end{array}$ & 330 & 43 & 48 & 170 \\
\hline$\alpha$-Chymotrypsin & $\begin{array}{c}\text { Tyr, Phe, } \\
\text { Trp, Val, } \\
\text { Ile, Leu }\end{array}$ & 434 & 81 & 76 & 221 \\
\hline Pepsin & $\begin{array}{l}\text { Tyr, Phe, } \\
\text { Trp, Leu }\end{array}$ & 324 & 41 & 50 & 189 \\
\hline Trypsin & Lys, Ary & 26 & 15 & 20 & 4 \\
\hline
\end{tabular}

explanation for the low antigenicity of peptides degraded by $\alpha$-chymotrypsin compared to other samples in immunological detection. Additionally, previous research ${ }^{29}$ has demonstrated that soluble peptides will continue to be enzymatically cleaved in solution, probably resulting in the destruction of epitopes, especially spatial epitopes in further enzymatic hydrolysis. Therefore, the antigenicity of some samples decreased. Fortunately, all peptides obtained by enzymatic hydrolysis could be specifically detected, though they were degraded into peptides. The result was of great significance for the detection of ancient silk fabric residues.

\section{Conclusions}

The degradation process of SF from B. mori and A. pernyi was studied following exposure to different enzymes. Although the cleavage sites were different, the four enzymes had similar effects on the structure and molecular-weight distribution of silk fibroin of the same species, probably owing to the accessibility of cleavage sites. An exhaustive analysis of the secondary structure revealed that the structural damage of $B$. mori SF focused on the $\beta$-sheet, while the destruction of $A$. pernyi SF focused on the $\alpha$-helix. The immunoassay results indicated that all samples were able to bind to the corresponding diagnostic antibodies by an antigen-antibody reaction without crossreactivity. Further analysis showed that alkaline proteinase and pepsin were more suitable for the degradation of SF because the enzyme-treated SF proteins consistently exhibited higher immunoreactivity to diagnostic antibodies due to the retention of epitopes on the SF peptides surface. These results provide insight into the possible degradation behavior of SF from different species. Meanwhile, the diagnostic antibodies can be further applied in the field of microdetection of silk fibroin.

\section{Acknowledgements}

Financial support was provided by the National Natural Science Foundation of China (51603188), the Public Technology Research Plan of Zhejiang Province, China (2016C33175), the Outstanding Young Research Program of Science and Technology for the Protection of Cultural Relics (2015-294), and the Special Funds from the Administration of Cultural Heritage of Zhejiang Province (2017014). 


\section{Supporting Information}

This material is available free of charge on the Web at http:// www.jsac.or.jp/analsci/.

\section{References}

1. J. Li, F. Cai, X. Ye, J. Liang, J. Li, M. Wu, D. Zhao, Z. Jiang, Z. You, and B. Zhong, J. Proteome Res., 2017, 16, 2495.

2. J. Glausiusz, Nature, 2009, 462, 574 .

3. W. Patrick, S. Hans, and P. Angelika, J. Agric. Food Chem., 2009, 57, 8399.

4. H. Mai, Y. Yang, I. Abuduresule, W. Li, X. Hu, and C. Wang, Sci. Rep., 2016, 6, 18939.

5. E. Karpova, V. Vasiliev, V. Mamatyuk, N. Polosmak, and L. Kundo, J. Archaeol. Sci., 2016, 70, 15.

6. M. A. Koperska, D. Pawcenis, J. Bagniuk, M. M. Zaitz, M. Missori, T. Łojewski, and J. Łojewska, Polym. Degrad. Stabil., 2014, 105, 185.

7. M. E.-S. Osman, A. A. E.-N. El-Shaphy, D. A. Meligy, and M. M. Ayid, Int. J. Conserv. Sci., 2014, 5, 295.

8. K. Sterflinger and F. Pinzari, Environ. Microbiol., 2012, 14, 559.

9. Q. Lu, B. Zhang, M. Li, B. Zuo, D. L. Kaplan, Y. Huang, and H. Zhu, Biomacromolecules, 2011, 12, 1080.

10. R. You, Y. Xu, Y. Liu, X. Li, and M. Li, Biomed. Mater, 2014, 10, 015003.

11. T. Wongpinyochit, B. F. Johnston, and F. P. Seib, ACS Biomater. Sci. Eng., 2018, 4, 942.

12. K. Numata, P. Cebe, and D. L. Kaplan, Biomaterials, 2010, 31,2926
13. E. S. Sashina, A. M. Bochek, N. P. Novoselov, and D. A. Kirichenko, Russ. J. Appl. Chem., 2006, 79, 869.

14. Q. You, M. Liu, Y. Liu, H. Zheng, Z. Hu, Y. Zhou, and B. Wang, ACS Sens., 2017, 2, 569.

15. T. Tsumuraya, T. Sato, M. Hirama, and I. Fujii, Anal. Chem., 2018, 90, 7318.

16. J. Gu, C. Xu, M. Li, B. Chen, Y. Shang, H. Zheng, Y. Zhou, Z. Hu, Z. Peng, and B. Wang, Anal. Sci., 2019, 35, 175.

17. M. Timms, R. Steel, and J. Vine, Drug Test. Anal., 2016, 8, 164.

18. S. Ling, Z. Qi, D. P. Knight, Z. Shao, and X. Chen, Biomacromolecules, 2011, 12, 3344.

19. G. Fang, S. Sapru, S. Behera, J. Yao, Z. Shao, S. C. Kundu, and X. Chen, J. Mater. Chem. B, 2016, 4, 4337.

20. K. Tanaka, N. Kajiyama, K. Ishikura, S. Waga, A. Kikuchi, K. Ohtomo, T. Takagi, and S. Mizuno, Biochim. Biophys. Acta, 1999, 1432, 92.

21. S. Inoue, K. Tanaka, F. Arisaka, S. Kimura, K. Ohtomo, and S. Mizuno, J. Biol. Chem., 2000, 275, 40517.

22. Q. You, Q. Li, H. Zheng, Z. Hu, Y. Zhou, and B. Wang, J. Agric. Food Chem., 2017, 65, 7805.

23. L. Hedstrom, Chem. Rev., 2003, 34, 4501.

24. I. Siddiqui and Q. Husain, Colloid. Surf. B, 2019, 173, 733.

25. S. Ha, H. S. Gracz, A. E. Tonelli, and S. M. Hudson, Biomacromolecules, 2005, 6, 2563.

26. C. Guo, J. Zhang, J. S. Jordan, X. Wang, R. W. Henning, and J. L. Yarger, Biomacromolecules, 2018, 19, 906.

27. Y. Wang, J. Wen, B. Peng, B. Hu, X. Chen, and Z. Shao, Biomacromolecules, 2018, 19, 1999.

28. D. J. Barlow, M. S. Edwards, and J. M. Thornton, Nature, 1986, 322, 747.

29. T. Arai, G. Freddi, R. Innocenti, and M. Tsukada, J. Appl. Polym. Sci., 2004, 91, 2383. 